\title{
Suppression of torsades de pointes by atropine
}

\author{
Hanno L Tan, Arthur A M Wilde, Ron J G Peters
}

\begin{abstract}
A 67 year old woman with a history of chronic atrial fibrillation presented with asthma cardiale. She took no medication and there was no family history of long QT syndrome. She was treated with furosemide, nitroprusside, acenocoumarol, and digoxin. Two days later excessively prolonged RR intervals, which were terminated by escape beats with a right bundle branch block morphology, suggested impending total AV block. There was also severe QT (0.48 s) and QTc (0.56) interval prolongation with bizarre inverted $T U$ waves and multifocal premature ventricular complexes within the $U$ waves. The patient experienced angina pectoris followed by episodes of torsades de pointes, which were interpreted as the result of bradycardia, and the bradycardia as the result of high grade AV block induced by increased vagal tone caused by ischaemia in the presence of digoxin intoxication (serum digoxin was $2.5 \mu \mathrm{g} / \mathrm{l}$ ). Subsequent atropine infusion sped up the ventricular rate and shortened the QT $(0.39)$ and QTc (0.51) intervals. Digoxin was replaced by metoprolol to control ventricular rate and angina pectoris. Within days, QT and QTc intervals became normal and the $U$ waves disappeared. Neither torsades de pointes nor angina pectoris recurred. Based on a review of the literature, it is suggested that
\end{abstract}

Accepted for publication 9 September 1997

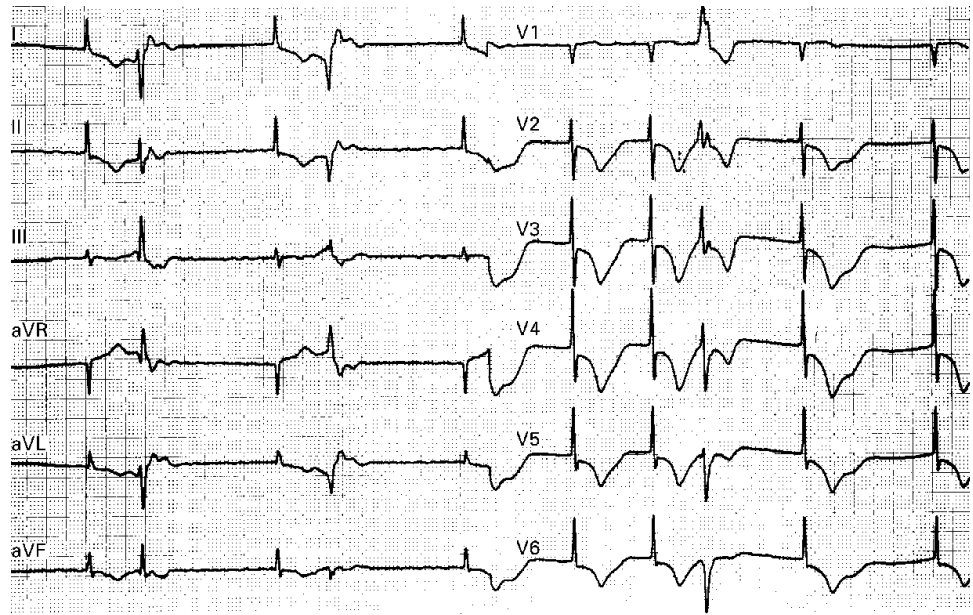

Figure 1 ECG two days after admission, after digoxin, in the presence of angina pectoris. Note bradycardia (50-60 beats/min) and severe $Q T$ prolongation with bizarre inverted $T U$ waves and frequent premature ventricular complexes appearing within the $U$ waves. $Q T$ (of the 6th beat) $0.60 \mathrm{~s}, Q T c 0.57$. the electrophysiological mechanism of this effect is not only an increase of the heart rate, but also a direct action of muscarinic receptor antagonism on Purkinje cells and ventricular refractoriness. (Heart 1998;79:99-100)

Keywords: torsades de pointes; long QT syndrome; atropine; AV block

Excessive QT interval prolongation (long QT syndrome) is an increasingly recognised cause of malignant ventricular tachyarrhythmias, including torsades de pointes. Various mechanisms may cause the net increase of inward transmembrane ion currents underlying torsades de pointes. ${ }^{1}$ In congenital long QT syndrome, torsades de pointes usually follows from increased adrenergic tone; in acquired long QT syndrome, torsades de pointes is associated with bradycardia. Torsades de pointes has been reported during AV block associated bradycardia. $^{2}$ This report demonstrates torsades de pointes suppression by atropine in a patient with bradycardia secondary to high grade AV block.

\section{Case report}

A 67 year old woman with a history of chronic atrial fibrillation was admitted for asthma cardiale. She took no medication. There was no family history of long QT syndrome. The ECG showed atrial fibrillation with a ventricular rate of $150-180$ beats/min without ischaemia. The QT interval was $0.25 \mathrm{~s}$ and the rate corrected (Bazett) QT interval (QTc) 0.45. Laboratory analysis showed normokalaemia and minimal myocardial damage (creatine kinase MB $8.4 \mu \mathrm{g} / 1$ exceeded our laboratory's normal level of 7). We administered furosemide, nitroprusside, acenocoumarol, and digoxin. Ventricular rate slowed to $80-90$ beats $/ \mathrm{min}$. However, after two days, excessively prolonged $\mathrm{RR}$ intervals, which were terminated by escape beats with a right bundle branch block morphology, suggested impending total AV block. Furthermore, there was a severe QT (0.48 s) and QTc (0.56) interval prolongation with bizarre inverted TU waves and multifocal premature ventricular complexes (PVCs) appearing within the U waves. Shortly thereafter the patient experienced angina pectoris with a further slowing of ventricular rate ( $50-60$ beats $/ \mathrm{min})$ and prolongation of QT (0.60) and QTc (0.57) intervals 


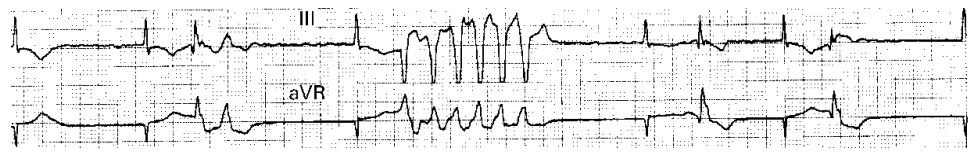

Figure 2 ECG shortly after that in fig 1 shows an episode of non-sustained torsades de pointes. Initiation of the tachycardia shows a typical short-long-short RR interval sequence (the 3 rd beat is followed after a short pause by the 4th beat, which is followed after a long pause by the 5th beat, which is followed after a short pause by the 6th beat, which constitutes the first beat of the tachycardia).

(fig 1). This was followed by episodes of torsades de pointes (fig 2). We interpreted the torsades de pointes as the result of bradycardia, and the bradycardia as the result of high grade $\mathrm{AV}$ block induced by increased vagal tone caused by ischaemia in the presence of digoxin intoxication (serum digoxin was $2.5 \mu \mathrm{g} / \mathrm{l}$ ).

Subsequent atropine infusion sped up the ventricular rate to $100-110$ beats $/ \mathrm{min}$ and shortened the QT (0.39) and QTc (0.51) intervals within the next hours. We replaced digoxin by metoprolol to control ventricular rate and angina pectoris. Within days, QT and QTc intervals became normal and the $U$ waves disappeared. Neither torsades de pointes nor angina pectoris recurred.

\section{Discussion}

The effectiveness of atropine in suppressing torsades de pointes in this patient while speeding up her heart rate supports our hypothesis that her tachyarrhythmia was caused by bradycardia associated with high grade $\mathrm{AV}$ block secondary to an increased vagal tone. Bradycardia prolongs QT intervals by reducing the repolarising $\mathrm{Na}^{+} / \mathrm{K}^{+}$pump activity and causing more complete decay of the repolarising delayed rectifier $\mathrm{K}^{+}$current. ${ }^{1}$ Furthermore, delayed adaptation of QT interval duration to sudden heart rate slowing may facilitate ventricular tachyarrhythmias. ${ }^{3}$ On the other hand, muscarinic receptor stimulation has direct (independent of AV conduction slowing) electrophysiological effects on Purkinje ${ }^{4}$ and ventricular cells ${ }^{5}$ that may facilitate torsades de pointes. While torsades de pointes may be initiated by early afterdepolarisations (EADs) arising from Purkinje fibres and spreading to ventricular myocardium, ${ }^{1}$ acetylcholine may facilitate EADs by lengthening Purkinje fibre action potentials, ${ }^{4}$ whereas atropine should counteract EADs by shortening ventricular refractory periods. ${ }^{5}$ Indeed, acetylcholine injection caused QT prolongation and torsades de pointes in patients with congenital long QT syndrome. ${ }^{6}$ Thus, in our patient, atropine may have suppressed torsades de pointes by speeding up the heart rate as well as by its direct effect on ventricular refractoriness.

1 Tan HL, Hou CJY, Lauer MR, Sung RJ. Electrophysiologic mechanisms of te long mechanisms of the long QT interval syndromes
torsade de pointes. Ann Intern Med 1995;122:701-14.

2 Kurita T, Ohe T, Marui N, Aihara N, Takaki H, Kamakura $\mathrm{S}$, et al. Bradycardia-induced abnormal QT prolongation in patients with complete atrioventricular block with torsades de pointes. Am F Cardiol 1992;69:628-33.

3 Jeron A, Würth A, Faber TS, Zehender M. Delayed adaptation of ventricular repolarisation after conversion from atrial fibrillation - a proarrhythmic risk factor? [abstract] Circulation 1996;94:I245.

4 Lipsius SL, Gibbons WR. Acetylcholine lengthens action potentials of sheep cardiac Purkinje fibers. Am 7 Physiol potentials of sheep car

5 Prystowsky EN, Jackman WM, Rinkenberger RL, Heger JJ, Zipes DP. Effect of autonomic blockade on ventricular refractoriness and atrioventricular nodal conduction in humans: evidence supporting a direct cholinergic action on ventricular muscle refractoriness. Circ Res 1981;49:511-18.

6 Aizawa Y, Washizuka T, Igarashi Y, Kitazawa H, Chinushi $\mathrm{M}$, Abe A, et al. Acetylcholine-induced prolongation of the QT interval in idiopathic long QT syndrome. Am 7 Cardiol 1996;77:879-82. 